Bundesgesundheitsbl $2020 \cdot 63: 113-121$ https://doi.org/10.1007/s00103-019-03057-y Online publiziert: 13 . November 2019 (c) Der/die Autor(en) 2019

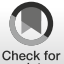

Matthias Raspe ${ }^{1} \cdot$ Peter Koch $^{2} \cdot$ Max Zilezinski ${ }^{3} \cdot K^{2}$ evin Schulte ${ }^{4}$ Diane Bitzinger ${ }^{5}$. Ulrike Gaiser ${ }^{6}$. Andreas Hammerschmidt ${ }^{7} \cdot$ Ralf Köhnlein $^{8}$. Julian Puppe ${ }^{9}$. Florian Tress ${ }^{10}$. Theodor Uden ${ }^{11}$. Albert Nienhaus ${ }^{2,12}$

'Medizinische Klinik m. S. Infektiologie und Pneumologie, Charité - Universitätsmedizin Berlin, Humboldt-Universität zu Berlin, Berlin, Deutschland; ${ }^{2}$ Institut für Versorgungsforschung in der Dermatologie und bei Pflegeberufen, Universitätsklinikum Hamburg-Eppendorf, Hamburg, Deutschland; ${ }^{3}$ Geschäftsbereich Pflegedirektion - Pflegewissenschaft, Core-Team III Delirmanagement und Demenzpflege, Charité - Universitätsmedizin Berlin, Berlin, Deutschland; ${ }^{4}$ Klinik für Innere Medizin IV mit dem Schwerpunkt Nieren- und Hochdruckkrankheiten, Universitätsklinikum Schleswig-Holstein, Campus Kiel, Kiel, Deutschland; ${ }^{5}$ Klinik für Anästhesiologie, Universitätsklinikum Regensburg, Regensburg, Deutschland; ${ }^{6}$ Klinik für Kinderneurologie und Sozialpädiatrie, Kinderzentrum Maulbronn gGmbH, Maulbronn, Deutschland; ${ }^{7}$ Klinik für Orthopädie und Unfallchirurgie, KRH Klinikum Lehrte, Lehrte, Deutschland; ${ }^{8}$ Präventionsdienst, Berufsgenossenschaft für Gesundheitsdienst und Wohlfahrtspflege (BGW), Karlsruhe, Deutschland; ${ }^{9}$ Klinik und Poliklinik für Frauenheilkunde und Geburtshilfe, Uniklinik Köln, Köln, Deutschland; ${ }^{10}$ Klinik für Gefäßchirurgie, Universitätsklinik der Paracelsus Medizinischen Privatuniversität Klinikum Nürnberg, Standort Süd, Nürnberg, Deutschland;

"Zentrum für Kinderheilkunde und Jugendmedizin, Medizinische Hochschule Hannover, Hannover, Deutschland; ${ }^{12}$ Abteilung Arbeitsmedizin, Gefahrstoffe und Gesundheitswissenschaften (AGG), Berufsgenossenschaft für Gesundheitsdienst und Wohlfahrtspflege (BGW), Hamburg, Deutschland

\section{Arbeitsbedingungen und Gesundheitszustand junger Ärzte und professionell Pflegender in deutschen Krankenhäusern}

\section{Einleitung}

Der medizinisch-wissenschaftliche Fortschritt sowie der demografische Wandel haben die Bezahlbarkeit von solidarisch finanzierten und hoch entwickelten Gesundheitswesen $\mathrm{zu}$ einer großen gesellschaftlichen Herausforderung gemacht. In Deutschland hat diese Entwicklung zu einer Ökonomisierung geführt, welche als entscheidende und viel diskutierte Größe neben die primäre Patientenorientierung in das System getreten ist [1, $2]$.

Die Autoren M. Raspe und P. Koch teilen sich die Erstautorenschaft.

Hinweis zu geschlechterneutraler Sprache Aus Gründen der leichteren Lesbarkeit wird in der vorliegenden Arbeit überwiegend die gewohnte männliche Sprachform verwendet. Dies impliziert jedoch keine Benachteiligung des weiblichen Geschlechts, sondern soll im Sinne der sprachlichen Vereinfachung als geschlechtsneutral zu verstehen sein. Ärzten und professionell Pflegenden, aber auch anderen Berufsgruppen in deutschen Krankenhäusern werden diese Entwicklungen vor allem in Form einer hohen Arbeitsverdichtung erlebt [3-5]. Unterschiedliche Erhebungen aus jüngerer Zeit legen eine gesundheitliche Gefährdung durch diese Arbeitsbedingungen für Ärzte [6-13] und Pflegende $[14,15]$ nahe.

Die Verantwortung für die Gesundheit von Arbeitnehmern wird neben ihnen selbst, den Arbeitgebern und der Politik in relevantem Umfang von den Unfallversicherungen getragen. Im Sozialgesetzbuch (SGB) VII definiert der Gesetzgeber deren Aufgabe als, „mit allen geeigneten Mitteln Arbeitsunfälle und Berufskrankheiten sowie arbeitsbedingte Gesundheitsgefahren zu verhüten “ [16]. Hierzu gehören explizit auch psychische Gesundheitsgefahren [17].

Die Berufsgenossenschaft für $\mathrm{Ge}$ sundheitsdienst und Wohlfahrtspflege
Von jungen Angestellten - primär
(BGW) versichert über 8,4 Mio. Arbeitnehmer im Gesundheitswesen. Ihrem gesetzlichen Auftrag folgend, Gesundheitsgefährdungen aktiv zu erfassen, hat die BGW die Schirmherrschaft dieser interdisziplinären und interprofessionellen Erhebung unter jungen Angestellten in der stationären Patientenversorgung übernommen, die zur Klärung folgender Fragen beitragen soll:

1. Welchen Belastungen mit welcher Ausprägung sind junge Angestellte aktuell ausgesetzt?

2. Welchen Einfluss nehmen diese Belastungsfaktoren auf ihren Gesundheitszustand und auf die von ihnen erbrachte Patientenversorgung?

3. Welche Verbesserungsbedarfe sehen junge Angestellte als besonders wirkungsvoll?

\section{Methoden}

Im September 2017 wurde im Auftrag der BGW bei jungen Angestellten eine bun- 
desweite randomisierte Datenerhebung durchgeführt, mit deren unabhängiger Planung und Durchführung das Institut für Versorgungsforschung in der Dermatologie und bei Pflegeberufen am Universitätsklinikum Hamburg-Eppendorf betraut wurde. Der Feldzugang für diese anonyme Querschnittserhebung erfolgte anhand der Mitgliederdatenbanken der beteiligten Berufsverbände bzw. Fachgesellschaften. Kooperationspartner dieses Projektes waren sieben medizinische Verbände bzw. Gesellschaften sowie ein Berufsverband der Pflege (Marburger Bund, Hartmannbund, Deutsche Gesellschaft für Innere Medizin, Berufsverband Deutscher Internisten, Deutsche Gesellschaft für Kinder- und Jugendmedizin, Deutsche Gesellschaft für Gynäkologie und Geburtshilfe, Deutsche Gesellschaft für Anästhesiologie und Intensivmedizin sowie der Deutsche Berufsverband für Pflegeberufe).

Da es sich bei dieser Untersuchung um eine anonyme Befragung handelte, war keine Beratung durch eine Ethikkommission bzw. deren Votum notwendig.

Bei der Studienpopulation handelte es sich um junge Ärzte und junge Beschäftigte aus der Pflege ( $\leq 35$ Jahre), die im Krankenhaus arbeiteten und maximal 6 Jahre Berufserfahrung hatten. Vor Erhebungsbeginn wurde über die Gesellschaften/Verbände ein Flyer an die Mitglieder versandt. Auf der Startseite der Onlinebefragung wurden wesentliche Informationen zur Studie aufgeführt. Insbesondere wurden das Studienziel, die Freiwilligkeit einer Teilnahme, die Wahrung der Anonymität, die Konformität mit Bestimmungen des Bundesdatenschutzes, die Ausfüllzeit des Fragebogens und die Einwilligung zur Teilnahme durch Ausfüllen des Fragebogens beschrieben. Die Onlinebefragung wurde mit der Befragungssoftware EFS Survey von Questback/Unipark durchgeführt (Sicherheitsanforderungen nach ISO 27001 auf Basis des IT-Grundschutzes). Der Fragebogen wurde im Vorfeld in einem Pretest von 40 Teilnehmern getestet. Als Incentives wurden Tickets für den Hauptstadtkongress Medizin und Gesundheit und den Deutschen Pflegetag verlost. Insgesamt erhielten 10.162 Mitglieder (6362 Ärzte, 3800
Beschäftigte aus der Pflege) via E-Mail eine Einladung zu der Onlinebefragung. Zwei und vier Wochen nach der Einladung erfolgte für alle Eingeladenen per E-Mail eine Erinnerung zur Studienteilnahme. Um ein ausgewogenes Verhältnis von Pflegenden und Ärzten zu erreichen, wurde im Vorfeld geplant, genauso viele Ärzte einzuladen, wie die Gesamtanzahl der Pflegenden des Deutschen Berufsverbands für Pflegeberufe (DBfK) ergab, die laut Mitgliederdatenbank die Einschlusskriterien $\leq 35$ Jahre und stationäre Versorgung erfüllten (ein 1:1-Verhältnis von Ärzten zu Pflegenden in der Stichprobe wurde angestrebt). Für die Pflege erfolgte demnach eine Vollerhebung. Um die Gefahr eines Selektionsbias gering zu halten und eine gleiche Anzahl von Ärzten als Zielpopulation auszuwählen, erfolgte für die Ärzte unter Berücksichtigung der Gesamtanzahl der Mitglieder pro Gesellschaft bzw. Verband eine proportional geschichtete Randomisierung. Die Randomisierung wurde im Studienzentrum anhand von laufenden Nummern über die Randomisierungsfunktion in SPSS durchgeführt. Die Nummern wurden dann an die Geschäftsstellen der beteiligten Fachgesellschaften/Berufsverbände weitergegeben, welche wiederum die ausgewählten Mitglieder zur Teilnahme einluden. Irrtümlicherweise wurden bei einer medizinischen Fachgesellschaft alle Mitglieder eingeladen, die die Einschlusskriterien erfüllten. Dies führte neben einer unterschiedlichen Responserate beider Berufsgruppen zusätzlich zu einem unausgewogenen Verhältnis zuungunsten der Pflege. Auf eine nachfolgende Reduzierung der Anzahl der teilnehmenden Ärzte wurde aus statistischen Gründen verzichtet.

Psychosoziale Faktoren wurden anhand der Kurzversion des branchenunabhängigen Effort-Reward-Imbalance(ERI-)Fragebogens erhoben [18, 19]. Das Arbeitsstressmodell basiert auf der Annahme, dass Beschäftigte für ihre Leistung (Effort) gesellschaftlich definierte Belohnungen (Reward) erhalten. Anhand der Skala Leistung sowie der Skala Belohnung (Subskalen: Anerkennung, Gehalt/Karrieremobilität, Arbeitsplatzsicherheit) werden die Leistungen und die Belohnungen ins Verhältnis zueinander gesetzt. Eine Effort-RewardImbalance wurde für einen ERI-Ratio über 1 definiert. Ein Überengagement (Overcommitment) wurde für den Wertebereich des obersten Terzils der Verteilung definiert. Als weitere psychosoziale Einflussgröße wurden Häufigkeiten des Erlebens von verbalen bzw. körperlichen Aggressionen durch Patienten in den Fragebogen aufgenommen $(1 \times \mathrm{im} \mathrm{Jahr}$, $4 \times$ im Jahr, $1 \times$ wöchentlich, täglich).

Um die Qualität der interprofessionellen Zusammenarbeit zwischen ärztlichem und pflegerischem Personal zu erheben, sind Skalen eines standardisierten Fragebogens zur Arbeitssituation von Ärzten bzw. professionell Pflegenden zum Einsatz gekommen [20].

Der subjektive Gesundheitszustand und das Burn-out-Risiko wurden als Gesundheitsoutcomes anhand des standardisierten Copenhagen Psychosocial Questionnaire (COPSOQ-Fragebogen) erhoben [21]. Zur Messung der subjektiven Versorgungsqualität wurde auf die von Loerbroks et al. [22] validierte deutschsprachige Version des Originalinstruments für Ärzte zurückgegriffen [23]. Die entsprechende Skala für professionell Pflegende wurde aus der originalen Skala für Ärzte abgeleitet. Dabei wurden zwei von sechs Items entsprechend angepasst. Beide Skalen wiesen gute interne Konsistenzen auf (Cronbachs Alpha: 0,80 (Ärzte) bzw. 0,77 (Pflege)).

Zur Ermittlung der Verbesserungsbedarfe wurden in der Befragung 29 verschiedene Themen präsentiert, die sieben verschiedenen Feldern zugeordnet waren (Zeit, Familie und Beruf, Zusammenarbeit, Belohnungen, Führung, Arbeitsorganisation/Mittel, Strukturelles). Beim Rating der verschiedenen Items ging es darum, den auf den Arbeitsplatz bezogenen aktuellen subjektiven Bedarf der Studienteilnehmer zu erfassen.

Bivariate Analysen wurden anhand des Chi ${ }^{2}$-Tests, des t-Tests, des MannWhitney-U-Tests und der einfaktoriellen Varianzanalyse durchgeführt. Um Verzerrungen durch Confounding gering $\mathrm{zu}$ halten, wurden multivariate Verfahren angewandt. Für die Outcomes subjektiver Gesundheitszustand und Burn-out- 
Bundesgesundheitsbl 2020 -63:113-121 https://doi.org/10.1007/s00103-019-03057-y

(c) Der/die Autor(en) 2019

M. Raspe · P. Koch · M. Zilezinski · K. Schulte · D. Bitzinger · U. Gaiser · A. Hammerschmidt $\cdot$ R. Köhnlein · J. Puppe · F. Tress · T. Uden · A. Nienhaus

\section{Arbeitsbedingungen und Gesundheitszustand junger Ärzte und professionell Pflegender in deutschen Krankenhäusern}

\section{Zusammenfassung}

Hintergrund. Tief greifende Veränderungen im Gesundheitswesen haben für junge Angestellte in der stationären Patientenversorgung zu einem stark verdichteten Arbeitsalltag geführt. Vorarbeiten legen eine Gesundheitsgefährdung durch diese Arbeitsbedingungen nahe.

Ziel der Arbeit. Diese Studie hatte zum Ziel, mit einer interprofessionellen Erhebung aktuelle Belastungsfaktoren, deren Konsequenzen und subjektive Verbesserungsbedarfe zu untersuchen.

Methoden. Im September 2017 wurde eine anonymisierte Querschnittserhebung unter Ärzten und professionell Pflegenden bis $\leq 35$ Jahre und mit maximal 6 Jahren

Berufserfahrung in der stationären Patienten- versorgung durchgeführt. Der Feldzugang erfolgte über sieben teilnehmende Fachgesellschaften und Berufsverbände. Der elektronische Fragebogen enthielt überwiegend validierte Erhebungsinstrumente. Durch Regressionsmodelle wurden mögliche Störfaktoren in die Datenanalyse einbezogen. Ergebnisse. In die endgültige Analyse gingen 1060 komplette Fälle ein. Die Teilnahmequote lag bei $13 \%$. Es wurden u. a. zeitliche und psychosoziale Belastungsfaktoren mit überwiegend erheblicher Ausprägung festgestellt. Diese Belastungsfaktoren sind mit einem reduzierten Gesundheitszustand und erhöhtem Burn-out-Risiko sowie einer subjektiv schlechteren Versorgungsqualität assoziiert. Junge Ärzte wünschen sich weniger Dokumentation und eine Reduktion der Arbeitsverdichtung; junge Pflegende erwarten eine leistungsgerechte Bezahlung und festgelegte Personalschlüssel.

Diskussion. Aktuelle Arbeitsbedingungen stellen eine Gesundheitsgefährdung junger Angestellter in der stationären Patientenversorgung dar. Es müssen Rahmenbedingungen geschaffen werden, die den Leistungserbringern nachhaltig ein gesundes und effektives Arbeiten ermöglichen.

Schlüsselwörter

Ärzte · Pflegende · Belastungen · Gesundheit . Auswirkungen

\section{Working conditions and health status of young physicians and nurses in German hospitals}

\section{Abstract}

Background. Profound transformations in the German healthcare system lead to intense working conditions for young employees in inpatient care. Published data suggest associated health risks.

Objectives. This investigation aims to assess current stress factors, their consequences, and subjective measures for improvement. Methods. During September 2017, a crosssectional survey was conducted among physicians and nurses $\leq 35$ years of age and with work experience in inpatient care not exceeding six years. Field access was gained via seven scientific and professional societies.
The electronic questionnaire consisted of mainly validated instruments. Regression models included confounders into the final data analysis.

Results. Included in the final data analysis were 1060 complete cases. The overall response rate was $13 \%$. Different stress factors (e.g. time-related or psychosocial) were found at high levels. These stress factors were connected to reduced health and higher risk of burnout. Furthermore, they were associated with inferior perceived quality of care. Young physicians claimed less documentation requirements and less intense working conditions. Young nurses demanded fair salary and an appropriate nurse-patient allocation.

Conclusion. Today's working conditions in inpatient care are a threat to the health status of young employees. General conditions, which ensure a sustainable healthy and effective working environment, need to be ensured.

\section{Keywords}

Physicians · Nurses · Stress factors · Health .

Consequences
Risiko wurden multivariate lineare Regressionen gerechnet. Adjustiervariablen waren Alter, Geschlecht und Berufsgruppe. Als mögliche Einflussfaktoren wurden folgende Variablen in das Modell miteingeschlossen: ERI, Overcommitment, interprofessionelle Zusammenarbeit, verbale/körperliche Aggressionen, Einnahme von Medikamenten aufgrund von Arbeitsstress, Beschäftigungsverhältnis (befristet/unbefristet), Wochenarbeitszeit sowie weitere arbeitsbezogene Merkmale wie Nachtschichten und Wochenendarbeit. Anhand der StepwiseBackwards-Methode nach Hosmer und
Lemeshow wurden Variablen mit einem $p$-Wert von $>0,1$ schrittweise aus dem Modell eliminiert [24].

Für das Outcome subjektive Versorgungsqualität wurde aufgrund fehlender Normalverteilung multivariate logistische Regression gerechnet. Die Dichotomisierung der Variable erfolgte nach Loerbroks et al. anhand des 3. Terzils [22]. Anhand des Stepwise-BackwardsVerfahrens erfolgte hier ebenfalls die Variablenreduktion im Modell [24].

Bei allen Analysen wurde eine mögliche Interaktion von ERI und Overcommitment überprüft.
Fehlende Werte: In einer Sensitivitätsanalyse mit imputierten fehlenden Werten wurden die finalen Modelle überprüft. Da sich hinsichtlich der Assoziationsmaße dieselben Tendenzen zeigten, werden im Folgenden die Ergebnisse ohne multiple Imputationen präsentiert.

Alle Auswertungen erfolgten mit der Statistiksoftware SPSS 23.0.

\section{Ergebnisse}

Insgesamt nahmen 1337 Beschäftigte an der Onlinebefragung teil (dies entspricht allen Teilnehmern, die den Fragebogen 


\begin{tabular}{|c|c|c|c|c|}
\hline & \multirow{2}{*}{$\begin{array}{l}\text { Professionell Pflegende } \\
N=205(19,3 \%) \\
\%, x(S D)^{a}\end{array}$} & \multirow{2}{*}{$\begin{array}{l}\text { Ärzte } \\
N=855(80,7 \%) \\
\%, \times(S D)^{a}\end{array}$} & \multicolumn{2}{|c|}{$\begin{array}{l}\text { Gesamt } \\
N=1060(100 \%)\end{array}$} \\
\hline & & & $p$ & $(N) \%, x(S D)^{a}$ \\
\hline \multicolumn{5}{|l|}{ Geschlecht } \\
\hline Weiblich & $69,8 \%$ & $60,4 \%$ & \multirow[t]{2}{*}{0,001} & (659) $62,2 \%$ \\
\hline Männlich & $30,2 \%$ & $39,6 \%$ & & (401) $37,8 \%$ \\
\hline \multicolumn{5}{|l|}{ Alter } \\
\hline Gesamt & $26,5(3,1)$ & $30,8(2,4)$ & 0,001 & $29,9(3,03)$ \\
\hline \multicolumn{5}{|c|}{ Arbeitsvertrag (fehlend: $N=8$ ) } \\
\hline Befristet & $23,3 \%$ & $89,4 \%$ & \multirow[t]{2}{*}{$<0,001$} & (807) $76,7 \%$ \\
\hline Unbefristet & $76,7 \%$ & $10,6 \%$ & & (245) $23,3 \%$ \\
\hline \multicolumn{5}{|c|}{ Beschäftigungsverhältnis (fehlend: $N=6$ ) } \\
\hline Vollzeit & $70,0 \%$ & $90,7 \%$ & \multirow[t]{2}{*}{$<0,001$} & (914) $86,7 \%$ \\
\hline Teilzeit & $30,0 \%$ & $9,3 \%$ & & (140) $13,3 \%$ \\
\hline \multicolumn{5}{|c|}{ Einsatzorte (fehlend: $N=1$ ) } \\
\hline $\mathrm{ITS}^{\mathrm{b}} / \mathrm{IMC}^{\mathrm{c}}$ & $29,9 \%$ & $16,4 \%$ & \multirow[t]{4}{*}{$<0,001$} & (201) $19,0 \%$ \\
\hline $\mathrm{OP}$ & $0,5 \%$ & $49,4 \%$ & & (423) $39,9 \%$ \\
\hline Normalstation & $59,8 \%$ & $23,5 \%$ & & (323) $30,5 \%$ \\
\hline $\begin{array}{l}\text { Ambulanz/ } \\
\text { Notaufnahme }\end{array}$ & $9,8 \%$ & $10,8 \%$ & & (112) $10,6 \%$ \\
\hline \multicolumn{5}{|c|}{$\begin{array}{l}\text { ax (SD): Mittelwert (Standardabweichung) } \\
\text { bITS: Intensivstation } \\
\text { 'IMC: Intermediate Care }\end{array}$} \\
\hline
\end{tabular}

beendet hatten). Die Responserate lag bei $13 \%$ (Ärzte: 18,5\%, Pflegende: 7,5\%). Nach Anwendung der Einschlusskriterien verblieben 1060 Fälle für die Auswertung (Ärzte: 80,7\%, Pflegende: 19,3\%). Einen Überblick zu den Basisdaten dieser Erhebung gibt • Tab. 1.

\section{Belastungsfaktoren und der Gesundheitszustand}

Die tatsächliche Wochenarbeitszeit war unter Ärzten weitaus höher als unter den professionell Pflegenden ( $>48 \mathrm{~h}$ /Woche: $71 \%$ vs. $10 \%, p<0,001)$. Beiden Wochenenddiensten zeigten sich für die Beschäftigten der Pflege dagegen höhere Anteile als bei Ärzten ( $>2$ Wochenenddienste/ Monat: $21 \%$ vs. $13 \%, p<0,01$; • Tab. 2). Das Erleben verbaler und körperlicher Aggressionen durch Patienten trat insgesamt sehr häufig auf. Im Vergleich der Berufsgruppen zeigte sich, dass professionell Pflegende weitaus öfter körperliche bzw. verbale Aggressionen erlebten als das ärztliche Personal ( $74 \%$ vs. $34 \%$, $p<0,001$ bzw. $84 \%$ vs. $70 \%, p<0,001)$. Pflegende bewerteten die interprofessionelle Zusammenarbeit häufiger gering als

\section{Einflussfaktoren auf den Gesundheitszustand und Burn-out-Risiko}

In der multivariaten linearen Regression wurden verschiedene Faktoren gleichzeitig auf ihren Einfluss auf den subjektiven Gesundheitszustand und das Burn-out-Risiko untersucht (• Tab. 3). Als Faktoren mit statistisch signifikant positivem bzw. protektivem Einfluss ergaben sich die drei Dimensionen der ERI-Reward-Skala: Anerkennung: Beta $=1,2$, $95 \%$-KI: $\quad 0,38-1,95 \quad(\mathrm{Ge}-$ sundheitszustand)/Beta $=-1,5, \quad 95 \%$ KI: $\quad-2,22--0,80 \quad$ (Burn-out-Risiko), Karrieremobilität: Beta $=0,7,95 \%-K I$ : 0,10-1,39/Beta $=-0,8,95 \%-\mathrm{KI}:-1,34-$ $-0,19$ und Arbeitsplatzsicherheit: Be$\mathrm{ta}=1,0$, 95\%-KI: 0,13-1,83 (nur Gesundheitszustand). Eine bessere $\mathrm{Zu}$ sammenarbeit zwischen beiden Berufsgruppen hatte ebenfalls einen positiven Effekt auf den Gesundheitszustand (Be$\mathrm{ta}=1,6,95 \%-\mathrm{KI}: 0,73-2,44)$ und das Burn-out-Risiko (Beta $=-1,2,95 \%-\mathrm{KI}$ : -1,97--0,44). Faktoren mit statistisch signifikant negativem Einfluss auf den subjektiven Gesundheitszustand waren Wochenenddienste (Beta $=-4,2$, 95\%KI: -6,70--1,70), Overcommitment (Be$\mathrm{ta}=-1,5, \quad 95 \%-\mathrm{KI}:-1,85--1,23)$ und die Einnahme von Medikamenten (Be$\mathrm{ta}=-5,95 \%-\mathrm{KI}:-7,23--2,79)$. Für Burnout-Risiko waren Faktoren mit negativem bzw. verstärkendem Einfluss die Leistung (Effort; Beta $=0,8,95 \%$-KI: 0,22-1,35), die tatsächliche Arbeitszeit (Beta $=2,6,95 \%-\mathrm{KI}: \quad 0,71-4,56)$, körperliche Aggressionen (Beta $=2,1$, $95 \%-\mathrm{KI}:$ 0,33-3,81), Overcommitment (Beta $=2,0$, 95\%-KI: 1,77-2,33) sowie die Einnahme von Medikamenten (Beta $=3,9,95 \%$-KI: 1,90-5,88). Wurden die Effort- und Reward-Skalen in das ERIRatio transformiert, ergaben sich ebenfalls ungünstige Effekte in Bezug auf den Gesundheitszustand (Beta $=-5,6,95 \%$ KI: -8,09--3,05) und das Burn-outRisiko (Beta = 8,8, 95\%-KI: 6,57-11,12; Daten nicht in der Tabelle abgebildet). 
Tab. 2 Belastungsfaktoren und potenzielle Belastungsfolgen

Pflegende

$\%, X(S D)^{a}$

Ärzte

p

Gesamt

\begin{tabular}{|c|c|c|c|c|c|}
\hline & & $\%, X(S D)^{a}$ & $\%, X(S D)^{a}$ & & $\begin{array}{l}N(\%)^{b} \\
X(S D)^{a}\end{array}$ \\
\hline \multicolumn{6}{|l|}{ Belastungen durch Arbeitszeit } \\
\hline \multirow{2}{*}{$\begin{array}{l}\text { Tatsächliche Wochen- } \\
\text { arbeitszeit (fehlend: } N=4 \text { ) }\end{array}$} & $\leq 48 \mathrm{~h}$ & $90 \%$ & $29 \%$ & \multirow[t]{2}{*}{$<0,001$} & $430(41 \%)$ \\
\hline & $>48 \mathrm{~h}$ & $10 \%$ & $71 \%$ & & $626(59 \%)$ \\
\hline \multirow{2}{*}{$\begin{array}{l}\text { Wochenenddienste im Monat } \\
\text { (fehlend: } N=7 \text { ) }\end{array}$} & $\leq 2$ & $79 \%$ & $87 \%$ & \multirow[t]{2}{*}{$<0,010$} & $897(85 \%)$ \\
\hline & $>2$ & $21 \%$ & $13 \%$ & & $156(15 \%)$ \\
\hline \multicolumn{6}{|c|}{ Belastungen von Patientenseite } \\
\hline \multicolumn{2}{|c|}{$\begin{array}{l}\text { Verbale Aggressionen (mind. } 4 \times / J a h r ; \\
\text { fehlend: } N=15 \text { ) }\end{array}$} & $84 \%$ & $70 \%$ & $<0,001$ & $762(73 \%)$ \\
\hline \multicolumn{2}{|c|}{$\begin{array}{l}\text { Körperliche Aggressionen (mind. } 4 \times \text { / } \\
\text { Jahr; fehlend: } N=4 \text { ) }\end{array}$} & $74 \%$ & $34 \%$ & $<0,001$ & $442(42 \%)$ \\
\hline \multicolumn{6}{|c|}{ Weitere Belastungen } \\
\hline \multirow{3}{*}{$\begin{array}{l}\text { Interprofessionelle } \\
\text { Zusammenarbeit (fehlend: } \\
N=30 \text { ) }\end{array}$} & Gering & $24 \%$ & $9 \%$ & \multirow[t]{3}{*}{$<0,001$} & $123(12 \%)$ \\
\hline & Mittel & $61 \%$ & $62 \%$ & & $634(62 \%)$ \\
\hline & Hoch & $15 \%$ & $29 \%$ & & $273(27 \%)$ \\
\hline \multicolumn{2}{|c|}{$\begin{array}{l}\text { Effort-Reward-Imbalance Ratio }>1 \text { (feh- } \\
\text { lend: } N=38 \text { ) }\end{array}$} & $97 \%$ & $78 \%$ & $<0,001$ & $836(82 \%)$ \\
\hline \multicolumn{2}{|c|}{ Overcommitment (fehlend: $N=26$ ) } & $63 \%$ & $64 \%$ & 0,786 & $660(64 \%)$ \\
\hline \multicolumn{6}{|l|}{ Potenzielle Belastungsfolgen } \\
\hline \multicolumn{2}{|c|}{$\begin{array}{l}\text { Medikamente wg. Arbeitsstress (jemals; } \\
\text { fehlend: } N=3 \text { ) }\end{array}$} & $15 \%$ & $22 \%$ & 0,046 & $214(20 \%)$ \\
\hline \multicolumn{2}{|c|}{$\begin{array}{l}\text { Geringe Versorgungsqualität (fehlend: } \\
N=38 \text { ) }\end{array}$} & $59 \%$ & $31 \%$ & $<0,001$ & $373(37 \%)$ \\
\hline \multicolumn{2}{|l|}{ Subjektiver Gesundheitszustand } & $56,2(16,9)$ & $63,6(15,9)$ & $<0,001$ & $62,2(16,4)$ \\
\hline \multicolumn{2}{|l|}{ Burn-out (fehlend: $N=4$ ) } & $57,1(16,3)$ & $56,0(16,6)$ & 0,375 & $56,2(16,5)$ \\
\hline
\end{tabular}

\section{Einflussfaktoren auf die subjektive Versorgungsqualität}

Hinsichtlich der subjektiven Versorgungsqualität hatten Personen mit hohem ERI-Ratio (3. Terzil) ein 3-fach erhöhtes Odds Ratio (OR: 3,0, $95 \%$-KI: 2,00-4,62; - Tab. 4) und Personen aus der mittleren Gruppe (2. Terzil) ein 1,9-fach erhöhtes Odds Ratio (OR: 1,9, $95 \%$-KI: $1,28-2,85)$, eine geringe Versorgungsqualität zu empfinden wie Personen aus der niedrigsten Gruppe (1. Terzil). Für Beschäftigte mit niedriger Zusammenarbeit zeigte sich ein 2,6-fach erhöhtes OR (OR: 2,6, 95\%-KI: 1,52-4,32) und für diejenigen aus der mittleren Gruppe ein 1,8-fach erhöhtes Odds Ratio (OR: 1,8, $95 \%$-KI: 1,22-2,54) im Vergleich zur Referenzgruppe. Für häufiges Erleben von verbalen Aggressionen zeigte sich ein erhöhtes Odds Ratio von 1,5 (95\%-KI:
$1,00-2,14)$, eine geringe Versorgungsqualität zu empfinden. Für Personen, die auf einer Normalstation arbeiteten, wurde ein 1,7-fach erhöhtes Odds Ratio (OR: 1,7, 95\%-KI: 1,25-2,41) beobachtet, eine geringe Versorgungsqualität zu erleben.

\section{Verbesserungsbedarfe}

In $\bullet$ Tab. 5 sind von 29 Themen die fünf häufigsten Verbesserungsbedarfe mit hohem bzw. sehr hohem Bedarf aufgeführt. Für Ärzte zeigte sich auf den Plätzen 1 bis 3 ein Verbesserungspotenzial hinsichtlich des Dokumentationsaufwandes, der Weiterbildungsmöglichkeiten und der Arbeitsverdichtung. Für die Pflege ging es primär um leistungsgerechte Bezahlung, einen gesetzlich festgelegten Personalschlüssel sowie um die Arbeitsverdichtung. Für beide Berufsgruppen wurde hinsichtlich der fünf relevantesten Verbesserungsbedarfe eine Schnittmenge bezüglich des Dokumentationsaufwandes, der Arbeitsverdichtung sowie des Personalschlüssels beobachtet.

\section{Diskussion}

In dieser interdisziplinären und interprofessionellen Erhebung wurden Bestehen, Ausprägung und Einfluss aktueller Belastungsfaktoren bei jungen Ärzten und professionell Pflegenden in der stationären Patientenversorgung untersucht. Als Hauptbefund wurde eine Gesundheitsgefährdung unter den aktuellen Arbeitsbedingungen festgestellt.

\section{Aktuelle Belastungen im Arbeitsumfeld junger Angestellter}

Zeitliche Belastungsfaktoren waren vor allem unter Ärzten stark ausgeprägt und bestätigen das Ergebnis anderer aktueller Erhebungen. Im Marburger Bund Monitor 2017 wurde beispielsweise eine durchschnittliche Arbeitszeit von $51 \mathrm{~h}$ pro Woche für Ärzte ermittelt [4].

Verbale und körperliche Aggressionen, die von Patienten ausgehen, waren nach den Ergebnissen dieser Erhebung sehr häufig. Insgesamt wurden solche Aggressionen inklusive des Ungleichgewichts zuseiten professionell Pflegender in aktuellen Studien in ähnlich hohem Ausmaß beschrieben [3, 25-29]. Die hohen Prävalenzen von Aggressionen könnten anteilig auch durch eine zunehmende Sensibilisierung mit dem Thema erklärt werden. Welchen Anteil die aktuelle Versorgungssituation mit wenig Zeit und häufig zersplitterter und diskontinuierlicher Versorgung daran hat, müssen zukünftige Untersuchungen klären.

Im Vergleich mit COPSOQ-Referenzdaten (Beschäftigte aus Krankenhäusern bis 34 Jahre, gemittelt über den Zeitraum 2011 bis 2016, persönliche Mitteilung durch Dr. Matthias Nübling, Mitglied des Lenkungsausschusses COPSOQ International Network) ergaben sich für unser Studienkollektiv ungünstigere Ausprägungen in Bezug auf den subjektiven Gesundheitszustand (Ärzte/ Pflegende, aktuelle Studie vs. Referenz- 


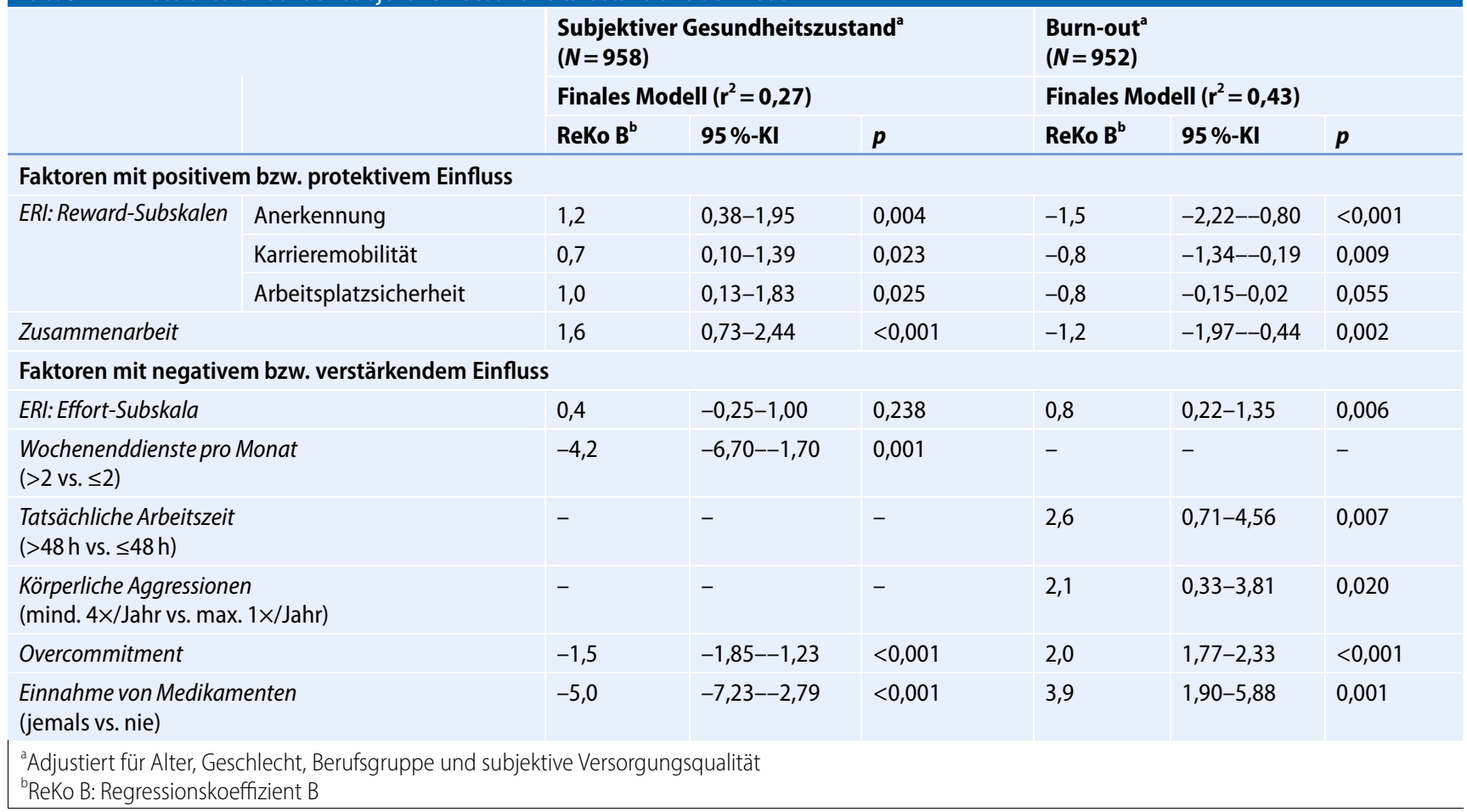

\section{Tab. 4 Einflussfaktoren auf die subjektive Versorgungsqualität}

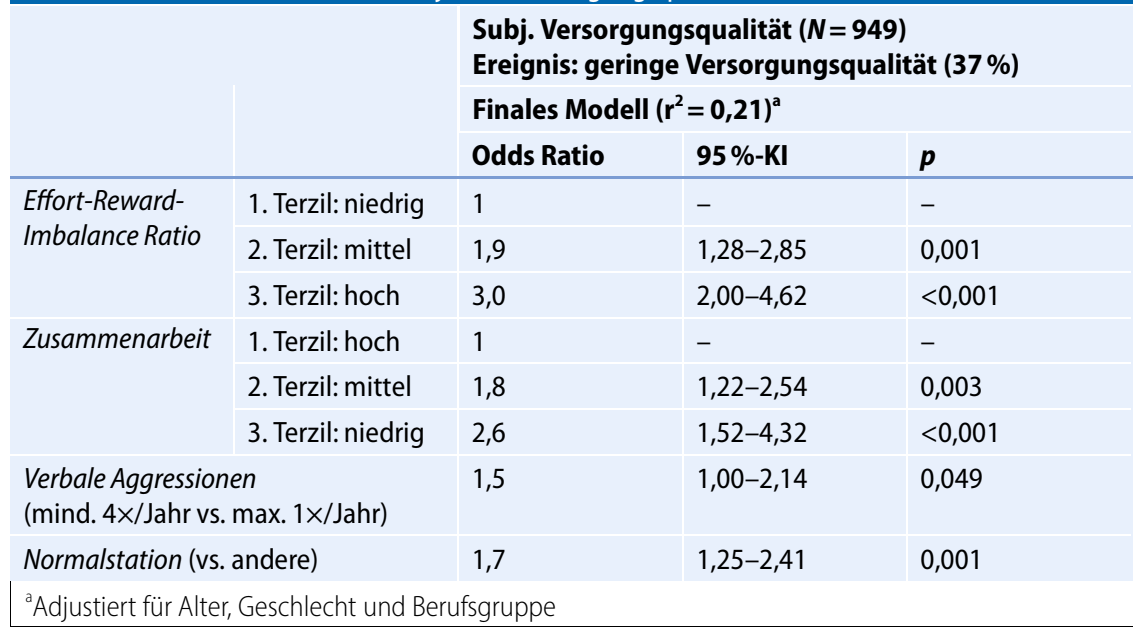

daten: $64 / 56$ vs. $80 / 73)$ und das Burnout-Risiko (56/57 vs. 54/53). Der Ausschluss der 35-Jährigen in unserer Studie ergab dieselben Mittelwerte, eventuell könnte der Vergleich aber auch durch unterschiedliche Erhebungsverfahren oder Selektionsbias verzerrt sein. Der Präsident des Weltärztebundes warnte kürzlich eindringlich vor der hohen Burn-out-Prävalenz. Fast die Hälfte der zehn Millionen weltweit arbeitenden Ärzte würden Symptome von Burnout zeigen [30]. In einer aktuellen US- amerikanischen Untersuchung gaben $45 \%$ der jungen Ärzte Symptome von Burn-out an [31]. Für Pflegende variierte die Burn-out-Rate nach den 2012 veröffentlichten Befunden einer großen internationalen Studie mit über 60.000 untersuchten Pflegekräften in Europa und den USA zwischen $10 \%$ in den Niederlanden bis $\mathrm{zu} 78 \%$ in Griechenland [32]. Die Ausprägung psychosozialer Arbeitsbelastung und einer erhöhten Neigung zum Überengagement war unter den Teilnehmern dieser Befra- gung im Vergleich zu inter-/nationalen Untersuchungen sehr hoch (ERI-Ratio Mittelwert $\overline{\mathrm{X}}$ : 1,6 bei Pflegenden und $\overline{\mathrm{X}}: 1,3$ bei Ärzten dieser Untersuchung; im Vergleich dazu beispielswiese ERIRatio bei französischen bzw. deutschen Pflegenden 0,7 (ORSOSA-Studie mit 2194 Pflegenden in Frankreich [33]) bzw. 0,6 (NEXT-Studie mit 1054 Pflegenden in Deutschland [34]); ERI-Ratio in einer populationsbasierten deutschen Stichprobe unter 1342 Personen 0,6 [35]; für eine Übersicht unterschiedlicher nationaler/internationaler Vergleichswerte siehe [36]). Ähnlich ausgeprägt war die ERI-Ratio bei aktuellen Erhebungen unter jungen Ärzten unterschiedlicher Fachbereiche in Deutschland mit 1,4-1,9 [6-9] und bei Ärzten in Griechenland mit 1,6 [37].

Zuletzt berichtete ein relevanter Anteil der jungen Angestellten von der Einnahme von Medikamenten aufgrund von Arbeitsstress. Die bedenkliche Einnahme von Substanzen/Suchtmitteln mit entspannender Wirkung unter Ärzten ist nicht selten. In einer kürzlich veröffentlichten Studie wurde beispielsweise von knapp einem Viertel der befragten deutschen Ärzte aus der stationären 


\begin{tabular}{|l|l|l|}
\hline Tab. 5 & Subjektive Verbesserungsbedarfe & \\
\hline Platz & Verbesserungsbedarfe der Ärzte & Anteil (\%) \\
\hline 1 & Verringerung des Dokumentationsaufwands & 88 \\
\hline 2 & Persönliche, strukturierte Weiterbildungsmöglichkeiten & 86 \\
\hline 3 & Verringerung der Arbeitsverdichtung & 86 \\
\hline 4 & Gesetzlich festgelegte Personalschlüssel & 84 \\
\hline 5 & Weniger Einfluss der Ökonomie auffachliche Entscheidungen & 84 \\
\hline Platz & Verbesserungsbedarfe professionell Pflegender & Anteil (\%) \\
\hline 1 & Leistungsgerechte Bezahlung & 93 \\
\hline 2 & Gesetzlich festgelegte Personalschlüssel & 92 \\
3 & Verringerung der Arbeitsverdichtung & 85 \\
4 & Verringerung des Dokumentationsaufwands & 83 \\
\hline 5 & Verbindlichkeit von Absprachen & 82 \\
\hline
\end{tabular}

Patientenversorgung ein bedenklicher Alkoholkonsum berichtet [38].

\section{Konsequenzen für den Gesund- heitszustand junger Angestellter und die Patientenversorgung}

Die drei Dimensionen der Belohnungsskala des Modells beruflicher Gratifikationskrisen wiesen in dieser Untersuchung einen positiven bzw. protektiven Einfluss auf den Gesundheitszustand und das Burn-out-Risiko auf. Andersherum war eine hohe Ausprägung psychosozialer Arbeitsbelastung (als Ausdruck eines hohen ERI-Ratio) mit einem negativen Einfluss auf die vorgenannten Gesundheitsqualitäten verbunden. Diese Assoziation wurde auch aus Voruntersuchungen berichtet, in denen vor allem eine hohe Prävalenz psychischer Erkrankungen wie Depressionen und Burn-out in Verbindung mit hoher psychosozialer Arbeitsbelastung beschrieben wurde [39-41]. Auch eine erhöhte Personalfluktuation am Arbeitsplatz wurde in Verbindung mit hoher psychosozialer Arbeitsbelastung berichtet $[42,43]$. Ein anderer protektiver Faktor war eine gute Zusammenarbeit zwischen Ärzten und Pflegenden. Internationale Studien konnten dazu passend zeigen, dass eine geringe interprofessionelle Zusammenarbeit eng mit geringer Arbeitszufriedenheit, Berufsaufgabe und Burnout assoziiert war [44-46]. Eine hohe Neigung zum Überengagement hat das Potenzial, Gratifikationskrisen zu verstärken, und war ein unabhängiger Risikofaktor für einen reduzierten Ge- sundheitszustand und hohes Burn-outRisiko. Das Gleiche galt für die Faktoren Wochenenddienste (reduzierter Gesundheitszustand), Arbeitszeit (mehr Burn-out-Risiko), körperliche Aggressionen (mehr Burn-out-Risiko) und die Einnahme von Medikamenten (reduzierter Gesundheitszustand und mehr Burn-out-Risiko). Zusammenfassend zeigen die oben genannten Ergebnisse dieser Untersuchung eine erhebliche Gesundheitsgefährdung unter aktuellen Arbeitsbedingungen.

Eine Assoziation mit einer als schlechter empfundenen Versorgungsqualität wurde in dieser Erhebung für hohe psychosoziale Arbeitsbelastung, schlechte Zusammenarbeit, häufige verbale Aggressionen von Patientenseite und das Arbeiten auf Normalstation nachgewiesen. Insbesondere die Assoziation von psychosozialer Arbeitsbelastung mit reduzierter subjektiver Versorgungsqualität wurde für Ärzte und Pflegende bereits in einer Vielzahl von Studien beschrieben [7, 14, 23, 32, 47-51]. Das Arbeiten auf Normalstation ist im Vergleich zum Arbeiten im Operationssaal in der Regel durch deutlich weniger Planbarkeit, weniger Personal, viele zeitgleich zu erledigende Aufgaben und eine insgesamt höhere Arbeitsverdichtung charakterisiert, sodass eine hier als geringer empfundene Versorgungsqualität schlüssig erscheint.

Subjektive Verbesserungsbedarfe junger Angestellter

Die Forderungen beider Berufsgruppen einschließlich ihrer unterschiedlichen Schwerpunkte sind aus der aktuellen politischen Diskussion weitestgehend bekannt. Während Pflegende an erster Stelle eine leistungsgerechte Bezahlung und festgelegte Personalschlüssel fordern, sind jungen Ärzten eine Reduktion der Dokumentationspflichten und Arbeitsverdichtung sowie eine strukturierte Weiterbildung wichtig.

\section{Limitationen}

Als Limitationen dieser Studie sind eine geringe Teilnahmequote, Disbalancen in der Stichprobe (Verteilung von Ärzten zu Pflegenden 4:1) und die Möglichkeit eines Selektionsbias zugunsten einer Teilnahme von unzufriedenen jungen Angestellten zu nennen. Ein Selektionsbias ist auch durch die eingeschlossenen Berufsverbände bzw. Fachgesellschaften möglich, die jeweils bei Pflegenden und Ärzten nur einen Teil der Arbeitnehmer mit ggf. besonderen Eigenschaften repräsentieren. Zudem ermöglicht die Querschnittsuntersuchung keine Rückschlüsse auf kausale UrsachenWirkungs-Beziehungen. Bezüglich der subjektiven Verbesserungsbedarfe ist einschränkend festzustellen, dass die Zustimmung zwischen den einzelnen Optionen zum Teil nur gering differiert. Um den Fragebogen schlank zu halten, sind Einzelskalen aus einem validierten Instrument selektiert worden (COPSOQ-Fragebogen). Es kann nicht ausgeschlossen werden, dass hierdurch die Validität dieser Einzelskalen eingeschränkt wurde.

\section{Fazit}

In Zeiten eines zunehmend unter Druck stehenden Arbeitens in der stationären Patientenversorgung und eines bestehenden Mangels an geeigneten Fachkräften zeigt diese Untersuchung aktuelle Belastungsfaktoren und assoziierte Beanspruchungen auf. Weiterhin werden die subjektiven Verbesserungsbedarfe junger Angestellter ermittelt. Kernbefund 


\section{Infobox 1 Zentrale Aussagen dieser Untersuchung}

- Das Arbeitsleben junger Ärzte und professionell Pflegender in der stationären Patientenversorgung ist durch erhebliche, wie z. B. zeitliche und psychosoziale Arbeitsbelastungen geprägt.

- Diese Belastungsfaktoren sind sowohl mit einem reduzierten Gesundheitszustand und Burn-out wie auch mit dem Empfinden einer geringen subjektiven Versorgungsqualität assoziiert.

- Anpassungen der Rahmenbedingungen sind gefordert, um den Leistungserbringern im Gesundheitswesen ein gesundes und effektives Arbeiten zu ermöglichen.

- Hierbei sind jungen Ärzten eine Reduktion von Dokumentationspflichten und Arbeitsverdichtung sowie eine strukturierte Weiterbildung wichtig. Junge Pflegende fordern primär eine leistungsgerechte Bezahlung und festgelegte Personalschlüssel.

dieser Erhebung ist eine erhebliche Gesundheitsgefährdung junger Angestellter in der stationären Patientenversorgung, die im Zusammenhang mit derzeitigen ungünstigen Arbeitsbedingungen steht (siehe auch $\bullet$ Infobox 1 für eine Zusammenfassung der zentralen Aussagen dieser Untersuchung). Aktuelle Rahmenbedingungen, die es den eigentlichen Leistungserbringern im Gesundheitssystem erschweren, gesund eine hochwertige Patientenversorgung zu gewährleisten, sollten dringlich angepasst werden. Da$\mathrm{zu}$ sind, neben den Betroffenen selbst, alle Parteien aufgerufen, die solche Rahmenbedingungen mitbestimmen - die Arbeitgeber, (Unfall-)Versicherungen und der Gesetzgeber.

\section{Korrespondenzadresse}

Albert Nienhaus
Abteilung Arbeitsmedizin, Gefahrstoffe
und Gesundheitswissenschaften (AGG),
Berufsgenossenschaft für Gesundheitsdienst
und Wohlfahrtspflege (BGW)
Pappelallee 35-37, 22089 Hamburg,
Deutschland
albert.nienhaus@bgw-online.de

\section{Einhaltung ethischer Richtlinien}

Interessenkonflikt. M. Raspe, P. Koch, M. Zilezinski, K. Schulte, D. Bitzinger, U. Gaiser, A. Hammerschmidt J. Puppe, F. Tress und T. Uden geben an, dass kein In- teressenkonflikt besteht. A. Nienhaus und R. Köhnlein erhielten von der Berufsgenossenschaft für Gesundheitsdienst und Wohlfahrtspflege (BGW) Gelder zur Durchführung dieses Forschungsprojekts. Die BGW hatte keinen Einfluss auf das Studiendesign und den Inhalt der Publikation.

Dieser Beitrag beinhaltet keine klinischen Studien an Menschen oder Tieren.

Open Access. Dieser Artikel wird unter der Creative Commons Namensnennung 4.0 International Lizenz (http://creativecommons.org/licenses/by/4.0/deed. de) veröffentlicht, welche die Nutzung, Vervielfältigung, Bearbeitung, Verbreitung und Wiedergabe in jeglichem Medium und Format erlaubt, sofern Sie den/die ursprünglichen Autor(en) und die Quelle ordnungsgemäßnennen, einen Linkzur Creative Commons Lizenz beifügen und angeben, ob Änderungen vorgenommen wurden.

\section{Literatur}

1. Deutscher Ethikrat (2016) Patientenwohl als ethischer Maßstab für das Krankenhaus

2. Leopoldina (2016) Zum Verhältnis von Medizin und Ökonomie im deutschen Gesundheitssystem - 8 Thesen zur Weiterentwicklung zum Wohle der Patienten und der Gesellschaft

3. Beerheide R (2017) Umfrage des Hartmannbundes: Junge Ärzte hadern mit Klinikalltag. Dtsch Arztebl Int 114:399-400

4. Marburger Bund (2017) MB-Monitor 2017. www. marburger-bund.de/bundesverband/themen/ marburger-bund-umfragen/mb-monitor-2017. Zugegriffen: 16. Juni 2019

5. Gröger A-C (2014) Eine Nachtschicht als Pfleger. Ärzte Zeitung. https://www.aerztezeitung.de/ politik_gesellschaft/pflege/article/875428/25jahren-heute-nachtschicht-pfleger.html. Zugegriffen: 16. Juni 2019

6. Raspe M, Müller-Marbach A, Schneider $M$, Siepmann T, Schulte K (2016) Work and training conditions of young German physicians in internal medicine. Results of a nationwide survey by young internists from the German Society of Internal Medicine and the German Professional Association of Internists. Dtsch Med Wochenschr 141:202-210

7. Raspe M, Vogelgesang A, Fendel J, Weiss C, Schulte K, Rolling T (2018) Work and Training Conditions of Young German Physicians in Internal Medicine-Results of a Second Nationwide Survey by Young Internists from the German Society of Internal Medicine and the German Professional Association of Internists. Dtsch Med Wochenschr 143:e42-e50

8. Arnold H, Meyer CP, Salem J, Raspe M, Struck JP, Borgmann $\mathrm{H}$ (2017) Work and training conditions of residents in urology in Germany : results of a 2015 nationwide survey by the German Society of Residents in Urology. Urologe A 56:1311-1319

9. Bitzinger D, Raspe M, Schulte K, Larmann J (2017) Evaluation der Arbeits- und Weiterbildungsbedingungen im Fachgebiet Anästhesiologie in Deutschland. Anästh Intensivmed 58:429-440

10. Biesalski AS, Franke C, Sturm D et al (2018) Nationwide survey of postgraduate medical training in clinical neurology. Nervenarzt. https:// doi.org/10.1007/s00115-018-0547-8

11. Thomas LR, Ripp JA, West CP (2018) Charter on physician well-being. JAMA 319:1541-1542
12. Stummer $\mathrm{H}$, Angerer $\mathrm{S}$, Jamnig $\mathrm{C}$, Nöhammer $\mathrm{E}$ (2018) Job satisfaction and intention to quitamong young physicians-evidence from Austria. Eur J Public Health 28:48

13. Gothe $H$, Köster AD, Storz P, Nolting HD, Häussler $B$ (2007) Job satisfaction among doctors. Dtsch Arztebl 104:A1394-A1399

14. Aiken LH, Clarke SP, Sloane DM, Sochalski J, Silber JH (2002) Hospital nurse staffing and patient mortality, nurse burnout, and job dissatisfaction. JAMA 288:1987-1993

15. Badura B, Schellschmidt H, Vetter C (2005) Fehlzeiten-Report 2004, Kapitel: Arbeitsbelastung und-beanspruchung bei Pflegepersonal in Europa - Ergebnisse der Next-Studie. Springer, Berlin Heidelberg

16. Sozialgesetzbuch online (2019) § 1 SGB VII Prävention, Rehabilitation, Entschädigung. www. sozialgesetzbuch-sgb.de/sgbvii/1.html. Zugegriffen: 16 . Juni 2019

17. Webseite der Berufsgenossenschaft für Gesundheitsdienst und Wohlfahrtspflege (2019) Psyche und Gesundheit - Wie Sie mit psychischer Belastung im Beruf umgehen können. www.bgw-online.de/DE/ArbeitssicherheitGesundheitsschutz/Psyche-und-Gesundheit/ Psyche-und-Gesundheit_node.html. Zugegriffen: 16 . Juni 2019

18. Leineweber $C$, Wege $N$, Westerlund $H$, Theorell $T$, Wahrendorf M, Siegrist J (2010) How valid is a short measure of effort-reward imbalance at work? A replication study from Sweden. Occup Environ Med 67:526-531

19. Siegrist J, Wege N, Puhlhofer F, Wahrendorf M (2009) A short generic measure of work stress in the era of globalization: effort-reward imbalance. Int Arch Occup Environ Health 82:1005-1013

20. Fischbeck S, Laubach W (2005) Arbeitssituation und Mitarbeiterzufriedenheit in einem Universitätsklinikum: Entwicklung von Messinstrumenten für ärztliches und pflegerisches Personal. Psychother Psychosom Med Psychol 55:305-314

21. Nübling M, Stößel U, Hasselhorn H-M, Michaelis M, Hofmann F (2006) Measuring psychological stress and strain at work: Evaluation of the COPSOQ Questionnaire in Germany. GMS Psychosoc Med 3:1-14

22. Loerbroks A, Weigl M, Li J, Angerer P (2016) Effortreward imbalance and perceived quality of patient care: a cross-sectional study among physicians in Germany. BMC Public Health 16:342

23. Shanafelt TD, Bradley KA, Wipf JE, Back AL (2002) Burnout and self-reported patient care in an internal medicine residency program. Ann Intern Med 136:358-367

24. Hosmer DW, Lemeshow S (2000) Applied logistic regression. Wiley \& Sons, New York

25. Schablon A, Wendeler D, Kozak A, Nienhaus A, Steinke $S$ (2018) Prevalence and consequences of aggression and violence towards nursing and care staff in Germany - a survey. Int J Environ Res Public Health 15(6):1274

26. Schablon A, Zeh A, Wendeler D et al (2012) Frequency and consequences of violence and aggression towards employees in the German healthcare and welfare system: a cross-sectional study. BmjOpen 2(5):e1420

27. Lindner $T$, Joachim $\mathrm{R}$, Bieberstein $\mathrm{S}$, Schiffer $\mathrm{H}$ Möckel M, Searle J (2015) Aggressives und herausforderndes Verhalten gegenüber dem KlinikpersonalAggressive and provocative behaviour towards medical staff. Notfall Rettungsmed 18:195-200 
28. Vorderwulbecke F, Feistle M, Mehring M, Schneider A, Linde K (2015) Aggression and violence against primary care physicians-a nationwide questionnaire survey. Dtsch Arztebl Int 112:159-165

29. Beerheide R (2018) Ärztemonitor 2018 - Zwischen Kümmerer und Burn-out. Dtsch Arztebl 115:1597-1598

30. Hil (2018) Weltärztebund-Warnung vor „BurnoutPandemie". Dtsch Arztebl 115:1844

31. Dyrbye L, Burke S, Hardeman R et al (2018) Association of Clinical Specialty With Symptoms of Burnout and Career Choice Regret Among US Resident Physicians. JAMA 320:1114-1130

32. Aiken LH, Sermeus W, Van Den Heede Ket al (2012) Patient safety, satisfaction, and quality of hospital care: cross sectional surveys of nurses and patients in 12 countries in Europe and the United States. BMJ 344:e1717

33. Herin F, Paris C, Levant A et al (2011) Links between nurses' organisational work environment and upper limb musculoskeletal symptoms: independently of effort-reward imbalance! The ORSOSA study. Pain 152:2006-2015

34. Li J, Galatsch M, Siegrist J, Muller BH, Hasselhorn HM, European NSG (2011) Reward frustration at work and intention to leave the nursing profession-prospective results from the European longitudinal NEXT study. Int J Nurs Stud 48:628-635

35. Nuebling $M$, Seidler A, Garthus-Niegel S et al (2013) The Gutenberg Health Study: measuring psychosocial factors at work and predicting health and work-related outcomes with the ERI and the COPSOQ questionnaire. BMC Public Health 13:538

36. Siegrist J, Li J, Montano D (2014) Psychometric properties of the Effort-Reward Imbalance Questionnaire. https://www.uniklinikduesseldorf.de/fileadmin/Fuer-Patienten-undBesucher/Kliniken-Zentren-Institute/Institute/ Institut fuer Medizinische Soziologie/Dateien/ ERI/PsychometricProperties.pdf. Zugegriffen: 16. Juni 2019

37. Msaouel P, Keramaris NC, Apostolopoulos AP et al (2012) The effort-reward imbalance questionnaire in Greek: translation, validation and psychometric properties in health professionals. Sangyo Eiseigaku Zasshi 54:119-130

38. Pforringer D, Mayer R, Meisinger C, Freuer D, Eyer F (2018) Health, risk behaviour and consumption of addictive substances among physicians-results of an online survey. J Occup Med Toxicol 13:27

39. Buddeberg-Fischer B, Klaghofer R, Stamm M, Siegrist J, Buddeberg C (2008) Work stress and reduced health in young physicians: prospective evidence from Swiss residents. Int Arch Occup Environ Health 82:31-38

40. Siegrist J, Starke D, Chandola T et al (2004) The measurement of effort-reward imbalance at work: European comparisons. Soc Sci Med 58:1483-1499

41. Van Vegchel N, De Jonge J, Bosma H, Schaufeli W (2005) Reviewing the effort-reward imbalance model: drawing up the balance of 45 empirical studies. Soc Sci Med 60:1117-1131

42. Soderberg $M$, Harenstam A, Rosengren $A$ et al (2014) Psychosocial work environment, job mobility and gender differences in turnover behaviour: a prospective study among the Swedish general population. BMC Public Health 14:605

43. Estryn-Behar M, Van Der Heijden BI, Fry C, Hasselhorn HM (2010) Longitudinal analysis of personal and work-related factors associated with turnover among nurses. Nurs Res 59:166-177
44. Aiken LH, Sloane DM, LakeET, Sochalski J, Weber AL (1999) Organization and outcomes of inpatient AIDS care. Med Care 37:760-772

45. Galletta M, Portoghese I, Battistelli A, Leiter MP (2013) The roles of unit leadership and nurse-physician collaboration on nursing turnover intention. J Adv Nurs 69:1771-1784

46. Zangaro GA, Soeken KL (2007) A meta-analysis of studies of nurses' job satisfaction. Res Nurs Health 30:445-458

47. Firth-Cozens J, Greenhalgh J (1997) Doctors' perceptions of the links between stress and lowered clinical care. Soc Sci Med 44:1017-1022

48. Fahrenkopf AM, Sectish TC, Barger LK et al (2008) Rates of medication errors among depressed and burnt out residents: prospective cohort study. BMJ 336:488-491

49. West CP, Huschka MM, Novotny PJ et al (2006) Association of perceived medical errors with resident distress and empathy: a prospective longitudinal study. JAMA 296:1071-1078

50. Klein J, Grosse Frie K, Blum K, von dem Knesebeck O (2011) Psychosocial stress at work and perceived quality of care among clinicians in surgery. BMC Health Serv Res 11:109

51. Panagioti M, Geraghty K, Johnson J et al (2018) Association between physician burnout and patient safety, professionalism, and patient satisfaction: a systematic review and metaanalysis. JAMA Intern Med 178:1317-1330 